\title{
An affine version of a theorem of Nagata
}

\author{
Gene Freudenburg
}

\begin{abstract}
Let $R$ be an affine $k$-domain over the field $k$. The paper's main result is that if $R$ admits a nontrivial embedding in a polynomial ring $K[s]$ for some field $K$ containing $k$, then $R$ can be embedded in a polynomial ring $F[t]$ which extends $R$ algebraically. This theorem can be applied to subrings of a ring which admits a nonzero locally nilpotent derivation. In this way, we obtain a concise new proof of the cancellation theorem for rings of transcendence degree one for fields of characteristic 0 .
\end{abstract}

\section{Introduction}

If $F \subset E$ are fields and $x \in E$, then the subfield of $E$ generated by $F$ and $x$ is denoted by $F(x)$. If $x$ is transcendental over $F$, then $F(x)$ is isomorphic to the field of rational functions in one variable over $F$, and we write $F(x) \cong F^{(1)}$. In his 1967 paper, Nagata [15] proved the following fundamental result for fields.

THEOREM 1.1 ([15, THEOREM 2] AND [17, THEOREM 5.2])

Let $k, K, L$ be fields such that

(a) $k \subset K$ and $k \subset L \subset K^{(1)}$;

(b) $K$ is finitely generated over $k$;

(c) $L \not \subset K$.

Then there exists a finite algebraic extension of the form $L \subset M^{(1)}$ for some field $M$ containing $k$.

This result extends the famous theorem of Lüroth, which asserts that if $k \subset L \subset$ $k(x)$ are fields with $k \neq L$ and $x$ transcendental over $k$, then there exists $y \in k(x)$ with $L=k(y)$. By combining the theorems of Lüroth and Nagata, we get an even stronger statement for fields of transcendence degree one over $k$ (see the Appendix).

We consider an analogous situation for integral domains. The polynomial ring in one variable $x$ over the field $F$ is denoted by $F[x]=F^{[1]}$. For the integral domain $R$, we seek criteria to determine when $R=F^{[1]}$ or when $R \subset F^{[1]}$ with $F^{[1]}$ algebraic over $R$. Our main result is Theorem 2.1, which may be regarded as an affine version of Nagata's theorem:

Kyoto Journal of Mathematics, Vol. 55, No. 3 (2015), 663-672

DOI $10.1215 / 21562261-3089136$, (C) 2015 by Kyoto University

Received May 23, 2014. Revised August 18, 2014. Accepted August 25, 2014.

2010 Mathematics Subject Classification: 13B25, 14R10. 
Let $k$ be a field, and let $R$ be an affine $k$-algebra. Suppose that there exists a field $K$ with $R \subset K^{[1]}$ and $R \not \subset K$. Then there exists a field $F$ and an algebraic extension $R \subset F^{[1]}$.

This result is of particular interest in the setting of locally nilpotent derivations, where we assume that the ground field $k$ is of characteristic 0 . If an integral $k$-domain $B$ admits a nonzero locally nilpotent derivation $D$, then $B \subset K[s]$, where $K$ is the field of fractions of the kernel of $D$ and $s$ is a local slice. Thus, any affine subalgebra $R \subset B$ not contained in the Makar-Limanov invariant of $B$ is isomorphic to a nontrivial subring of $F[s]$ for some field $F$, where $F[s]$ is algebraic over $R$.

For rings of transcendence degree one over $k$, Theorem 3.1 gives an even stronger conclusion.

Let $k$ be a field, and let $R$ be a k-algebra with $\operatorname{tr} \cdot \operatorname{deg}_{k} R=1$. Suppose that there exists a field $K$ with $R \subset K^{[1]}$ and $R \not \subset K$. Then $R$ is $k$-affine and there exists a field $F$ algebraic over $k$ with $R \subset F^{[1]}$. If $k$ is algebraically closed, then there exists $t \in \operatorname{frac}(R)$ with $R \subset k[t]$.

Abhyankar, Eakin, and Heinzer [1] proved that if $R, S$ are integral domains of transcendence degree one over a field $k$ such that the polynomial rings $R\left[x_{1}, \ldots\right.$, $\left.x_{n}\right]$ and $S\left[y_{1}, \ldots, y_{n}\right]$ are isomorphic $k$-algebras, then $R$ and $S$ are isomorphic. In Section 4, we apply Theorem 3.1, together with the well-known theorems of Seidenberg and Vasconcelos on derivations, to obtain a short proof of this result in the case in which $k$ is of characteristic 0. Makar-Limanov [14] gave a proof of this result for $k=\mathbb{C}$, and we follow his idea to use the Makar-Limanov invariant. Other proofs are given in [5] for perfect fields and in [6] for the case in which $k$ is algebraically closed.

\subsection{Background}

Lüroth's theorem was proved by Lüroth [13] for the field $k=\mathbb{C}$ in 1876 and for all fields by Steinitz [22] in 1910. One generalization states that if $k \subset L \subset$ $k\left(x_{1}, \ldots, x_{n}\right)$ and $L$ is of transcendence degree one over $k$, then $L=k(y)$. This was proved by Gordan [10] for $k=\mathbb{C}$ in 1887 and for all fields by Igusa [12] in 1951; other proofs appear in [15] and [20]. In 1894, Castelnuovo [2] showed that if $\mathbb{C} \subset L \subset \mathbb{C}\left(x_{1}, \ldots, x_{n}\right)$ and $L$ is of transcendence degree two over $\mathbb{C}$, then $L=\mathbb{C}\left(y_{1}, y_{2}\right)$. Castelnuovo's result does not extend to nonalgebraically closed ground fields or to fields $L$ of higher transcendence degree. An excellent account of ruled fields and their variants can be found in [17], including the theorem of Nagata [17, Theorem 5.2].

For polynomial rings, Evyatar and Zaks [7] showed that if $R$ is a PID and $k \subset R \subset k\left[x_{1}, \ldots, x_{n}\right]$, then $R=k^{[1]} ;$ Zaks [24] generalized this to the case in which $R$ is a Dedekind domain. Abhyankar, Eakin, and Heinzer [1, (2.5)] showed that if $k \subset R \subset k\left[x_{1}, \ldots, x_{n}\right]$ and $R$ is of transcendence degree one over $k$, then $R$ is isomorphic to a subring of $k^{[1]}$. Theorem 3.1 below generalizes these earlier results. It should be noted that Makar-Limanov [14, Lemma 14] proved a result equivalent to Theorem 3.1 for the field $k=\mathbb{C}$, which is stated in the language of locally nilpotent derivations (see also [5, Lemma 5.3] and [6, Lemma 4.2]). 
The case when $R$ is normal is of particular interest: If $k \subset R \subset k^{[n]}$ and $R$ is normal of transcendence degree one over $k$, then $R=k^{[1]}$. This was shown by Cohn [3, Proposition 2.1] for $n=1$, and in the stated form by Abhyankar, Eakin, and Heinzer [1, (2.6), (2.7)].

One generalization deals with the case where the field $k$ is replaced by a unique factorization domain (UFD). Abhyankar, Eakin, and Heinzer [1, Theorem 4.1] treat this case: If, for some integer $n \geq 1, A \subset D \subset A^{[n]}$ are UFDs such that the transcendence degree of $D$ over $A$ is one, then $D=A^{[1]}$. See also [19, Corollary 3.4] and [1, Proposition 4.8]. Connell and Zweibel [4, Theorem 4.1] present what they call "an affine version of Lüroth's theorem," namely: If $A$ is a UFD and $A \subset B \subset A[x]=A^{[1]}$ for a $\operatorname{ring} B$, then $\operatorname{frac}(B) \cap A[x]=A[v]$ for some $v \in A[x]$, where $\operatorname{frac}(B)$ denotes the field of fractions of $B$. The authors state that their result "is just an abstraction of what is proved in the proof of Theorem 2" in the paper of Formanek [8].

The Makar-Limanov invariant of a commutative ring (defined below) was introduced by Makar-Limanov in the mid-1990s, and he called it the ring of absolute constants. It is an important invariant in the study of affine rings, affine varieties, and their automorphisms.

\subsection{Preliminaries}

If $B$ is an integral domain, then $\operatorname{frac}(B)$ is the quotient field of $B$, and $B^{[n]}$ is the polynomial ring in $n$ variables over $B$. Given $f \in B, B_{f}$ denotes the localization $B[1 / f]$. The set of derivations $D: B \rightarrow B$ is $\operatorname{Der}(B)$.

If $A \subset B$ is a subring, then the transcendence degree of $B$ over $A$, denoted tr. $\operatorname{deg}_{A} B$, will mean the transcendence degree of $\operatorname{frac}(B)$ over $\operatorname{frac}(A)$. The set of elements of $B$ algebraic over $A$ is denoted by $\operatorname{Alg}_{A} B$.

Let $k$ be a field of characteristic zero, and let $B$ be an integral domain containing $k$. The set of $k$-derivations $D: B \rightarrow B$ is denoted by $\operatorname{Der}_{k}(B)$, and $D$ is said to be locally nilpotent if, for each $b \in B$, there exists $n \in \mathbb{N}$ with $D^{n} b=0$. The set of locally nilpotent derivations of $B$ is denoted by $\operatorname{LND}(B)$. If $D \in \operatorname{LND}(B)$ is nonzero and $A$ is the kernel of $D$, then $A$ is algebraically closed in $B$ and tr. $\operatorname{deg}_{A}(B)=1$.

The Makar-Limanov invariant of $B$ is the intersection of all kernels of locally nilpotent derivations of $B$, denoted $\operatorname{ML}(B)$. Note that $\operatorname{ML}(B)$ is a $k$-algebra which is algebraically closed in $B$, and note that any automorphism of $B$ maps $\operatorname{ML}(B)$ into itself.

An element $s \in B$ is a local slice of $D$ if $D^{2} s=0$ and $D s \neq 0$. Note that every nonzero element of $\operatorname{LND}(B)$ admits a local slice. If $s \in B$ is a local slice of $D$, then

$$
B_{D s}=A_{D s}[s]=\left(A_{D s}\right)^{[1]} .
$$

This implies the following property: If $D f \in f B$ for some $f \in B$, then $D f=0$. The reader is referred to [9] for further details regarding locally nilpotent derivations.

We also need the following. 
PROPOSITION 1.1 ([16, PROPOSITION 5.1.2])

Let $k$ be a field, and let $A$ be a commutative $k$-algebra. Then, for any field extension $L / k, A$ is finitely generated over $k$ if and only if $L \otimes_{k} A$ is finitely generated over $L$.

\section{Main theorem}

For a field $K$, the polynomial ring $K[s]=K^{[1]}$ is naturally $\mathbb{Z}$-graded over $K$, where $s$ is homogeneous of degree one. Let deg be the associated degree function in $s$ over $K$. A subring $R \subset K[s]$ is homogeneous if the $\mathbb{Z}$-grading restricts to $R$.

\section{LEMMA 2.1}

Suppose that $K$ is a field, and suppose that $R \subset K[s]=K^{[1]}$ is a homogeneous subring with $R \not \subset K$. Let $L=\operatorname{frac}(R) \cap K$, and let $\hat{L}, \hat{K}$ denote the algebraic closures of $L$ and $K$, respectively. Then there exist $c \in \hat{K}$ and integer $d \geq 1$ such that $R \subset \hat{L}\left[c s^{d}\right]$ and $\hat{L}\left[c s^{d}\right]$ is algebraic over $R$.

Proof

Define the integer

$$
d=\operatorname{gcd}\{\operatorname{deg} r \mid r \in R, r \neq 0\} .
$$

Let homogeneous $r \in R$ of positive degree be given. Then there exist $\kappa \in K$ and positive $e \in \mathbb{Z}$ with $r=\kappa s^{d e}$. Let $c \in \hat{K}$ be such that $c^{e}=\kappa$. Then $r=\left(c s^{d}\right)^{e}$.

If $\rho \in R$ is any other homogeneous element of positive degree, then $\rho=$ $\left(c^{\prime} s^{d}\right)^{e^{\prime}}$ for $c^{\prime} \in \hat{K}$ and positive $e^{\prime} \in \mathbb{Z}$. We have that

$$
\frac{r^{e^{\prime}}}{\rho^{e}}=\frac{\left(\left(c s^{d}\right)^{e}\right)^{e^{\prime}}}{\left(\left(c^{\prime} s^{d}\right)^{e^{\prime}}\right)^{e}}=\left(\frac{c}{c^{\prime}}\right)^{e e^{\prime}} \in L \quad \Rightarrow \quad \frac{c}{c^{\prime}} \in \hat{L} \quad \Rightarrow \quad \hat{L}\left[c^{\prime} s^{d}\right]=\hat{L}\left[c s^{d}\right] .
$$

It follows that $R \subset \hat{L}\left[c s^{d}\right]$.

\section{THEOREM 2.1}

Let $k$ be a field, and let $R$ be an affine $k$-algebra. Suppose that there exists a field $K$ with $R \subset K^{[1]}$ and $R \not \subset K$. Then there exist a field $F$ and an algebraic extension $R \subset F^{[1]}$.

Proof

Suppose that $R \subset K[s]=K^{[1]}$. For each $g \in K[s]$, let $\bar{g}$ denote the highest-degree homogeneous summand of $g$ as a polynomial in $s$. Define the set

$$
\bar{R}=\{\bar{r} \mid r \in R, r \neq 0\} .
$$

Then $k[\bar{R}]$ is a homogeneous subalgebra of $K[s]$ not contained in $K$.

By Lemma 2.1, if $L=\operatorname{frac}(k[\bar{R}]) \cap K$ and if $\hat{L}, \hat{K}$ are the algebraic closures of $L$ and $K$, respectively, then

$$
k[\bar{R}] \subset \hat{L}\left[c s^{d}\right] \quad(c \in \hat{K}, d \geq 1) .
$$


By hypothesis, there exist $w_{1}, \ldots, w_{m} \in R(m \geq 1)$ such that $R=k\left[w_{1}, \ldots\right.$, $\left.w_{m}\right]$. Given $i$, assume that $w_{i}=\sum_{j=0}^{n_{i}} c_{i j} s^{j}$, where $c_{i j} \in K$. Define $A \subset \hat{K}$ and $B \subset \hat{K}[s]$ by

$$
A=\hat{L}\left[c, c_{i j} \mid 1 \leq i \leq m, 0 \leq j \leq n_{i}\right] \quad \text { and } \quad B=A[s]=A^{[1]} .
$$

Then $R \subset B, A$ is finitely generated over $\hat{L}$, and the Jacobson radical of $A$ is trivial. Choose a maximal ideal $\mathfrak{m}$ of $A$ not containing $c$.

If $R \cap \mathfrak{m} B \neq(0)$, then let nonzero $r \in R \cap \mathfrak{m} B$ be given. Since $\mathfrak{m} B=\mathfrak{m}[s]$, we have $r=\sum_{0 \leq i \leq e} a_{i} s^{i}$, where $a_{i} \in \mathfrak{m}$ for each $i$. Note that $e \geq 1$, since $\hat{L} \cap \mathfrak{m}=(0)$. Therefore, by (2), there exist $\epsilon \geq 1$ and nonzero $\lambda \in \hat{L}$ such that

$$
\bar{r}=a_{e} s^{e}=\lambda\left(c s^{d}\right)^{\epsilon} .
$$

But then $c \in \mathfrak{m}$, a contradiction. Therefore, $R \cap \mathfrak{m} B=(0)$.

Let $\pi: B \rightarrow B / \mathfrak{m} B$ be the canonical surjection of $\hat{L}$-algebras, noting that

$$
B / \mathfrak{m} B=(A / \mathfrak{m} A)[\pi(s)]=\hat{L}^{[1]} .
$$

Since $\pi\left(c s^{d}\right)=\pi(c) \pi(s)^{d}$, where $\pi(c) \neq 0$, we see that $\left.\pi\right|_{R}$ is a degree-preserving isomorphism. It follows that $R$ is a subring of $\hat{L}^{[1]}$ via $\pi$.

It remains to show that $R$ and $\hat{L}^{[1]}$ have the same transcendence degree over $k$. Since $R \subset \hat{L}^{[1]}$, it will suffice to show that tr. $\operatorname{deg}_{k} \hat{L}^{[1]} \leq \operatorname{tr} \cdot \operatorname{deg}_{k} R$. By Lemma 2.1, we see that tr. $\operatorname{deg}_{k} \hat{L}^{[1]}=\operatorname{tr} . \operatorname{deg}_{k} k[\bar{R}]$, so it will suffice to show that tr.deg $k[\bar{R}] \leq$ tr. $\operatorname{deg}_{k} R$.

Let $n=\operatorname{dim}_{k} R$, and let $r_{1}, \ldots, r_{n+1} \in R$ be given. Then there exists a polynomial $h \in k\left[x_{1}, \ldots, x_{n+1}\right]=k^{[n+1]}$ with $h\left(r_{1}, \ldots, r_{n+1}\right)=0$. If $k\left[x_{1}, \ldots, x_{n+1}\right]$ is $\mathbb{Z}$-graded in such a way that each $x_{i}$ is homogeneous and the degree of $x_{i}$ is $\operatorname{deg} r_{i}$, then $H\left(\bar{r}_{1}, \ldots, \bar{r}_{n+1}\right)=0$, where $H$ is the highest-degree homogeneous summand of $h$. We have thus shown that any subset of $n+1$ elements in a generating set for $k[\bar{R}]$ is algebraically dependent over $k$. Therefore, the transcendence degree of $k[\bar{R}]$ over $k$ is at most $n$. This completes the proof of the theorem.

\section{Rings of transcendence degree one}

\section{THEOREM 3.1}

Let $k$ be a field, and let $R$ be a $k$-algebra with $\operatorname{tr} \cdot \operatorname{deg}_{k} R=1$. Suppose that there exists a field $K$ with $R \subset K^{[1]}$ and $R \not \subset K$. Then $R$ is $k$-affine and there exists a field $F$ algebraic over $k$ with $R \subset F^{[1]}$. If $k$ is algebraically closed, then there exists $t \in \operatorname{frac}(R)$ with $R \subset k[t]$.

Proof

Suppose that $R \subset K[s]=K^{[1]}$, and let deg be the associated degree function in $s$ over $K$.

Consider first the case in which $k$ is algebraically closed. The set

$$
\Sigma:=\{\operatorname{deg} w \mid w \in R, w \neq 0\} \subset \mathbb{N}
$$

is a semigroup and is therefore finitely generated as a semigroup. Let $w_{1}, \ldots, w_{m} \in$ $R$ be such that $\Sigma=\left\langle\operatorname{deg} w_{1}, \ldots, \operatorname{deg} w_{m}\right\rangle$, and define $S=k\left[w_{1}, \ldots, w_{m}\right] \subset R$. 
Then, given $v \in R$, there exists $u \in S \operatorname{such}$ that $\operatorname{deg} u=\operatorname{deg} v$. Assume that $\operatorname{deg} v \geq 1$.

As in the preceding proof, since $u$ and $v$ are algebraically dependent over $k, \bar{u}$ and $\bar{v}$ are also algebraically dependent over $k$. Since $u$ and $v$ have the same degree, there exists $P \in k[x, y]=k^{[2]}$ which is homogeneous relative to the standard $\mathbb{Z}$ grading of $k[x, y]$ such that $P(\bar{u}, \bar{v})=0$. Write $P(x, y)=\prod_{1 \leq i \leq \ell}\left(\alpha_{i} x+\beta_{i} y\right)$, where $\ell$ is a positive integer and $\alpha_{i}, \beta_{i} \in k^{*}(1 \leq i \leq \ell)$. Then $\alpha_{i} \bar{u}+\beta_{i} \bar{v}=0$ for some $i$. Therefore, $\operatorname{deg}\left(\alpha_{i} u+\beta_{i} v\right)<\operatorname{deg} v$ for some $i$. By induction on degrees, we can assume that $\alpha_{i} u+\beta_{i} v \in S$, which implies that $v \in S$, and $R=S$. Therefore, $R$ is finitely generated over $k$ when $k$ is algebraically closed.

For general $k$, let $\hat{k}$ and $\hat{K}$ denote the algebraic closures of $k$ and $K$, respectively. Set $\hat{R}=\hat{k} \otimes_{k} R$. Then $\operatorname{tr} \cdot \operatorname{deg}_{\hat{k}} \hat{R}=1, \hat{R} \subset \hat{K}^{[1]}$, and $\hat{R} \not \subset \hat{K}$. By what was shown above, we conclude that $\hat{R}$ is affine over $\hat{k}$. Therefore, Proposition 1.1 implies that $R$ is affine over $k$.

By Theorem 2.1, there exists a field $F$ algebraic over $k$ with $R \subset F^{[1]}$. If $k$ is algebraically closed, then $F=k$ and $k \subset R \subset k[s]$ for some $s$ transcendental over $k$. If $\mathcal{O}$ is the integral closure of $R$ in $\operatorname{frac}(R)$, then since $k[s]$ is integrally closed, we have that $k \subset R \subset \mathcal{O} \subset k[s]$. In this situation, it is known that $\mathcal{O}=k[\theta]$ for some $\theta \in k[s]$ (see [3, Proposition 2.1]).

\section{COROLLARY 3.1 (SEE [14, LEMMA 14])}

Let $k$ be an algebraically closed field of characteristic 0 , and let $B$ be a commutative $k$-domain. Given $r \in B$, if $r \notin \mathrm{ML}(B)$, then there exists $t \in \operatorname{frac}\left(\operatorname{Alg}_{k[r]} B\right)$ such that $\operatorname{Alg}_{k[r]} B \subset k[t]$.

\section{Proof}

By hypothesis, there exists $D \in \operatorname{LND}(B)$ with $D r \neq 0$. If $A=\operatorname{ker} D$ and $K=$ $\operatorname{frac}(A)$, then $K \otimes_{k} B=K^{[1]}$ by (1). We therefore have $\operatorname{Alg}_{k[r]} B \subset K^{[1]}$, and $r \notin K$. The result now follows by Theorem 3.1.

Makar-Limanov [14, p. 39] asked whether this result generalizes to rings of transcendence degree two. Let $k$ be an algebraically closed field of characteristic 0 , and let $B$ be a commutative $k$-domain. Given $x, y \in B$, does the implication

$$
\operatorname{Alg}_{k[x, y]} B \cap \operatorname{ML}(B)=k \quad \Rightarrow \quad \operatorname{Alg}_{k[x, y]} B \subset k^{[2]}
$$

hold?

\section{EXAMPLE 3.1}

Let $k$ and $K$ be fields with $k \subset K$, where $K=k[\alpha]$ is a simple algebraic extension of $k$, and $[K: k] \geq 2$. Define

$$
R=k[u, v] \subset K[s]=K^{[1]},
$$

where $u=\alpha s^{2}$ and $v=\alpha s^{3}$. Since $s=v / u$ and $\alpha=u^{3} / v^{2}$, we see that $\operatorname{frac}(R)=$ $K(s)$. If $R \subset k[t]$ for $t \in \operatorname{frac}(R)$, then $k(t)=\operatorname{frac}(R)=K(s)$, which is not possible. Therefore, the ring $R$ cannot be embedded in $k^{[1]}$. This shows that the 
hypothesis that the field $k$ is algebraically closed is necessary in the last statement of Theorem 3.1.

\section{EXAMPLE 3.2}

As an illustration of Corollary 3.1, let $k[x, y]=k^{[2]}$, and write $k[x, y]=\bigoplus_{i \geq 0} V_{i}$, where $V_{i}$ is the vector space of binary forms of degree $i$ over $k$. Define $D \in$ $\operatorname{LND}(k[x, y])$ by $D=x \frac{\partial}{\partial y}$. Then $D$ is linear, meaning that $D\left(V_{i}\right) \subset V_{i}$ for each $i$. Therefore, if $B=k\left[V_{2}, V_{3}\right]$, then $D$ restricts to $B$. Let $R$ be the algebraic closure of $k\left[y^{2}\right]$ in $B$, noting that $D\left(y^{2}\right) \neq 0$. Then $R=k\left[y^{2}, y^{3}\right]$ and $\operatorname{frac}(R)=k(y)$.

\section{Cancellation theorem for rings of transcendence degree one}

\subsection{Integral extensions and the conductor ideal} DEFINITION 4.1

Let $A$ and $B$ be integral domains with $A \subset B$. The conductor of $B$ in $A$ is

$$
\mathcal{C}_{A}(B)=\{a \in A \mid a B \subset A\} .
$$

If $\mathcal{O}$ is the integral closure of $A$ in $\operatorname{frac}(A)$, then the conductor ideal of $A$ is $\mathcal{C}_{A}(\mathcal{O})$.

Note that $\mathcal{C}_{A}(B)$ is an ideal of both $A$ and $B$, and is the largest ideal of $B$ contained in $A$. The following two properties of the conductor are easily verified:

(C.1) $\mathcal{C}_{A^{[n]}}\left(B^{[n]}\right)=\mathcal{C}_{A}(B) \cdot B^{[n]}$ for every $n \geq 0$;

(C.2) $D \mathcal{C}_{A}(B) \subset \mathcal{C}_{A}(B)$ for every $D \in \operatorname{Der}(B)$ restricting to $A$.

\section{LEMMA 4.1}

Let $k$ be a field, let $A$ be an integral domain containing $k$, and let $\mathfrak{C} \subset A$ be the conductor ideal of $A$. If $A$ is affine over $k$, then $\mathfrak{C} \neq(0)$.

\section{Proof}

Since $A$ is affine over $k$, its normalization $\mathcal{O}$ is also affine over $k$, and is finitely generated as an $A$-module (see [11, Chapter I, Theorem 3.9A]). Let $\left\{\omega_{1}, \ldots, \omega_{n}\right\}$ be a generating set for $\mathcal{O}$ as an $A$-module, and let nonzero $a \in A$ be such that $a \omega_{1}, \ldots, a \omega_{n} \in A$. Then $a \in \mathfrak{C}$.

THEOREM 4.1 (SEIDENBERG [21])

Let $A$ be a Noetherian integral domain containing $\mathbb{Q}$, and let $\mathcal{O}$ be the integral closure of $A$ in $\operatorname{frac}(A)$. Then every $D \in \operatorname{Der}(A)$ extends to $\mathcal{O}$.

THEOREM 4.2 (VASCONCELOS [23])

Let $A$ and $A^{\prime}$ be integral domains containing $\mathbb{Q}$ with $A \subset A^{\prime}$, where $A^{\prime}$ is an integral extension of $A$. If $D \in \operatorname{LND}(A)$ extends to $D^{\prime} \in \operatorname{Der}\left(A^{\prime}\right)$, then $D^{\prime} \in \operatorname{LND}\left(A^{\prime}\right)$. 


\subsection{The theorem of Abhyankar, Eakin, and Heinzer}

THEOREM 4.3 (SEE [1, (3.3)])

Let $k$ be a field, and let $R, S$ be integral $k$-domains of transcendence degree one over $k$. If $R^{[n]} \cong_{k} S^{[n]}$ for some $n \geq 0$, then $R \cong_{k} S$.

Proof

Characteristic $k=0$. Since $R$ is algebraically closed in $R^{[n]}$, we have that

$$
\operatorname{Alg}_{k}\left(R^{[n]}\right)=\operatorname{Alg}_{k}(R) .
$$

Let $\alpha: R^{[n]} \rightarrow S^{[n]}$ be an isomorphism of $k$-algebras. If $k^{\prime}=\operatorname{Alg}_{k}(R)$, then $\alpha\left(k^{\prime}\right)=$ $\operatorname{Alg}_{k}(S)$, since $S$ is algebraically closed in $S^{[n]}$. Therefore, identifying $k^{\prime}$ and $\alpha\left(k^{\prime}\right)$, we can view $R$ and $S$ as $k^{\prime}$-algebras, and $\alpha$ as a $k^{\prime}$-isomorphism. It thus suffices to assume that $k$ is algebraically closed in $R$.

Since $\operatorname{ML}\left(R^{[n]}\right) \subset \operatorname{ML}(R)$, we see that $\operatorname{ML}\left(R^{[n]}\right)$ is an algebraically closed subalgebra of $R$. Therefore, either $\operatorname{ML}\left(R^{[n]}\right)=R$ or $\operatorname{ML}\left(R^{[n]}\right)=k$.

Case 1: $\operatorname{ML}\left(R^{[n]}\right)=R$. In this case, we also must have $\operatorname{ML}\left(S^{[n]}\right)=S$. Since $\alpha$ maps the Makar-Limanov invariant onto itself, we conclude that $\alpha(R)=S$.

Case 2: $\operatorname{ML}\left(R^{[n]}\right)=k$. We will show that $R=k^{[1]}$ in this case. It suffices to assume that $k$ is an algebraically closed field: if $\hat{k}$ is the algebraic closure of $k$ and $\hat{R}=\hat{k} \otimes_{k} R$, then $\operatorname{ML}\left(\hat{R}^{[n]}\right)=\hat{k}$. If this implies $\hat{R}=\hat{k}^{[1]}$, then $R=k^{[1]}$. (All forms of the affine line over a perfect field are trivial; see [18].)

So assume that $k$ is algebraically closed. By hypothesis, there exists $D \in$ $\operatorname{LND}\left(R^{[n]}\right)$ with $D R \neq 0$. If $\mathcal{O}$ is the integral closure of $R$ in $\operatorname{frac}(R)$, then $\mathcal{O}^{[n]}$ is the integral closure of $R^{[n]}$ in $\operatorname{frac}\left(R^{[n]}\right)$. By property (C.1), if $\mathfrak{C}$ is the conductor ideal of $R$, then $\mathfrak{C} \cdot \mathcal{O}^{[n]}$ is the conductor ideal of $R^{[n]}$.

Let $s$ be a local slice of $D$, and let $K=\operatorname{frac}(\operatorname{ker} D)$. Then by $(1), R \subset K[s]$ and $R \not \subset K$. By Theorem 3.1, $R$ is $k$-affine, and there exists $t \in \operatorname{frac}(R)$ such that $\mathcal{O}=k[t]$. By the theorems of Seidenberg [21] and Vasconcelos [23], $D$ extends to a locally nilpotent derivation of $\mathcal{O}^{[n]}$; and by property $($ C.2 $), D\left(\mathfrak{C} \cdot \mathcal{O}^{[n]}\right) \subset \mathfrak{C} \cdot \mathcal{O}^{[n]}$.

By Lemma $4.1, \mathfrak{C} \neq 0$. Since $\mathfrak{C}$ is an ideal of $k[t]$, there exists a nonzero $h \in R$ with $\mathfrak{C}=h \cdot k[t]$. Thus, $\mathfrak{C} \cdot O^{[n]}=h \cdot \mathcal{O}^{[n]}$ and $D\left(h \cdot \mathcal{O}^{[n]}\right) \subset h \cdot \mathcal{O}^{[n]}$. Therefore, $D h=0$. If $h \notin k$, then $k[h] \subset \operatorname{ker} D$ implies that $R \subset \operatorname{ker} D$, which is not the case. Therefore, $h \in k^{*}$ and $R=k[t]$. By symmetry, $S=k^{[1]}$.

\section{REMARK 4.1}

The Makar-Limanov invariant can be defined for $k$-algebras over a field $k$ of any characteristic. This was done in [5], where it is defined to be the intersection of all invariant rings of actions of the additive group of $k$ on the ring. This is equivalent to the definition given above when the characteristic of $k$ is zero. Crachiola and Makar-Limanov [6, Corollary 3.2] use this approach to prove Theorem 4.3 in the case in which $k$ is algebraically closed. However, the theorems of Seidenberg [21] and Vasconcelos [23] are not available in positive characteristic, since they are valid for $\mathbb{Q}$-algebras. 


\section{Appendix}

Combining the theorems of Lüroth [13] and Nagata [15] gives the following corollary.

\section{COROLLARY A. 1}

Suppose that $k$ and $L$ are fields with $k \subset L$, where $k$ is algebraically closed, $L$ is finitely generated over $k$, and $\operatorname{tr} \cdot \operatorname{deg}_{k} L=1$. If there exists a field $E$ containing $k$ such that $L \subset E^{(1)}$ and $L \not \subset E$, then $L=k^{(1)}$.

\section{Proof}

Assume that $L \subset E(s)=E^{(1)}$. Let $\alpha_{1}, \ldots, \alpha_{n} \in L$ be such that $L=k\left(\alpha_{1}, \ldots, \alpha_{n}\right)$. Choose $f_{i}(s), g_{i}(s) \in E[s]$ such that $\alpha_{i}=f_{i} / g_{i}$, and let $K$ be the subfield of $E$ generated by the coefficients of $f_{i}$ and $g_{i}, 1 \leq i \leq n$. Then $K$ is finitely generated over $k$, and $L \subset K(s)$. By Nagata's theorem [15], there exists a finite algebraic extension $L \subset M^{(1)}$ for some field $M$ containing $k$. Since the transcendence degree of $L$ over $k$ is one, we see that $M$ is algebraic over $k$, that is, $M=k$. The corollary now follows by Lüroth's theorem [13].

We conclude by asking if the analogue of Theorem 2.1 holds for Laurent polynomial rings. Let $k$ be a field, and let $R$ be an affine $k$-algebra. Suppose that there exists a field $K$ with $R \subset K^{[ \pm 1]}$ and $R \not \subset K$. Does it follow that there exist a field $F$ and an algebraic extension $R \subset F^{[ \pm 1]}$ ?

Acknowledgments. The author wishes to thank Daniel Daigle of the University of Ottawa and Neena Gupta of the Indian Statistical Institute. Their comments on an earlier version of this paper led to a number of improvements.

\section{References}

[1] S. S. Abhyankar, W. Heinzer, and P. Eakin, On the uniqueness of the coefficient ring in a polynomial ring, J. Algebra 23 (1972), 310-342. MR 0306173.

[2] G. Castelnuovo, Sulla razionalità delle involuzioni piane, Math. Ann. 44 (1894), 125-155. MR 1510838. DOI 10.1007/BF01446977.

[3] P. M. Cohn, Subalgebras of free associative algebras, Proc. Lond. Math. Soc. (3) 14 (1964), 618-632. MR 0167504.

[4] E. Connell and J. Zweibel, Subrings invariant under polynomial maps, Houston J. Math. 20 (1994), 175-185. MR 1283269.

[5] A. J. Crachiola, On the AK invariant of certain domains, Ph.D. thesis, Wayne State University, Detroit, Mich., 2004. MR 2705802.

[6] A. J. Crachiola and L. Makar-Limanov, On the rigidity of small domains, J. Algebra 284 (2005), 1-12. MR 2115001. DOI 10.1016/j.jalgebra.2004.09.015. 
[7] A. Evyatar and A. Zaks, Rings of polynomials, Proc. Amer. Math. Soc. 25 (1970), 559-562. MR 0258820.

[8] E. Formanek, Observations about the Jacobian conjecture, Houston J. Math. 20 (1994), 369-380. MR 1287981.

[9] G. Freudenburg, Algebraic Theory of Locally Nilpotent Derivations, Encyclopaedia Math. Sci. 136, Springer, Berlin, 2006. MR 2259515.

[10] P. Gordan, Über biquadratische Gleichungen, Math. Ann. 29 (1887), 318-326. MR 1510417. DOI 10.1007/BF01447726.

[11] R. Hartshorne, Algebraic Geometry, Grad. Texts in Math. 52, Springer, New York, 1977. MR 0463157.

[12] J. Igusa, On a theorem of Lueroth, Mem. Coll. Sci. Univ. Kyoto Ser. A Math. 26 (1951), 251-253. MR 0048860.

[13] J. Lüroth, Beweis eines Satzes über rationale Curven, Math. Ann. 9 (1876), 163-165. MR 1509855. DOI 10.1007/BF01443371.

[14] L. Makar-Limanov, Locally nilpotent derivations, a new ring invariant and applications, Lecture notes, Bar-Ilan University, 1998, http://www.math.wayne.edu/ $\operatorname{lml} /$ (accessed 1 August 2014).

[15] M. Nagata, A theorem on valuation rings and its applications, Nagoya Math. J. 29 (1967), 85-91. MR 0207688.

[16] A. Nowicki, Polynomial Derivations and Their Rings of Constants, Uniwersytet Mikolaja Kopernika, Toruń, 1994. MR 2553232.

[17] J. Ohm, On ruled fields, Sém. Théor. Nombres, Bordeaux (2) 1 (1989), 27-49. MR 1050263.

[18] P. Russell, Forms of the affine line and its additive group, Pacific J. Math. 32 (1970), 527-539. MR 0265367.

[19] P. Russell and A. Sathaye, On finding and cancelling variables in $k[X, Y, Z]$, J. Algebra 57 (1979), 151-166. MR 0533106.

DOI 10.1016/0021-8693(79)90214-X.

[20] P. Samuel, Some remarks on Lüroth's theorem, Mem. Coll. Sci. Univ. Kyoto Ser. A Math. 27 (1953), 223-224. MR 0058251.

[21] A. Seidenberg, Derivations and integral closure, Pacific J. Math. 16 (1966), 167-173. MR 0188247.

[22] E. Steinitz, Algebraische Theorie der Körper, J. Reine Angew. Math. 137 (1910), 167-309.

[23] W. V. Vasconcelos, Derivations of commutative noetherian rings, Math. Z. 112 (1969), 229-233. MR 0254034.

[24] A. Zaks, Dedekind subrings of $k\left[x_{1}, \ldots, x_{n}\right]$ are rings of polynomials, Israel J. Math. 9 (1971), 285-289. MR 0280471.

Department of Mathematics, Western Michigan University, Kalamazoo, Michigan 49008, USA; gene.freudenburg@wmich.edu 